\title{
The impact of technologies on minimally invasive therapy
}

This issue of Surgical Endoscopy contains a new Technology Section set up by the two Editors in Chief with Dr. A. Melzer as the Technical Editor in charge of this Section. The primary objective is to review existing and emerging technology relating the whole spectrum of minimally invasive therapy or minimal access therapy. However, the preparation of the first Technology Section raised various issues relating to definitions, skills versus technology, co-operation between research clinicians and scientists/technologists and the anticipated future multidisciplinary therapeutic approach by disease-related treatment groups forming a coherent team of specialists from various disciplines working in close co-operation rather than as separate disciplines, with obvious gain in terms of the appropriate intervention in the individual case and improved patient outcome. The emergence of tomographic endosurgery covered in this issue exemplifies this approach which we must adopt as we approach the next millennium.

\section{Definitions}

Some ten years ago Wickham and Fitzpatrick introduced the phrase 'Minimally Invasive Therapy' (MIT) [1]. Although it lacks semantic accuracy and carries connotations of increased safety [2], this terminology has gained widespread acceptance and, indeed, it describes the essential goal of these endoscopic surgical and radiological interventions, i.e., the reduction of the operative traumatic insult without compromise of the therapeutic benefit. Since then, there has been considerable debate on alternative terminology such as minimal access therapy (MAT). Contention is avoided if the two terms MIT and MAT are considered to be synonymous with each comprising:

- minimally invasive surgery (MIS) or minimal access surgery (MAS) or endoscopic surgery (ES)

- interventional flexible endoscopy (EFE)

- interventional radiology (IR)

The importance of identification of these three components of MIT/MAT lies in the increasing combined use of two or all of these components in specific MIT/MAT treatment strategies for complex disorders, e.g., ES with EFE in the laparoscopic extraction of ductal calculi and ultrasound guided laparoscopic cryotherapy for hepatic metastases [3].

The combination of real time tomographic imaging, interventional radiology with frameless stereotactic navigation and endoscopic surgery has lead to the increasing use of image guided therapy or surgery (IGT/IGS). Although descriptive, this terminology does not distinguish between surface viewing by the endoscope and tomographic imaging by fluoroscopy, ultrasound, CT and MRI. The essence of this exciting new MIT approach is the combination of surface viewing of the operative field with near real time tomographic imaging of the operative tissue volume. The benefit of this dual imaging approach is increased precision of the therapeutic effect especially when frameless stereotaxis is used, thereby allowing further reduction in the size of the instruments and endoscopes, and the operative insult during the intervention. The current popular terminology for this evolving dual approach is image guided endoscopic microsurgery (IEM) [4] although the current imaging systems do not, as yet, provide a resolution higher than $1 \mathrm{~mm}^{3}$. Alternative terms include tomographic endoscopic microsurgery or, simply tomographic endosurgery (TES). IEM (or TES) represents the next phase in the reduction of the operative traumatic insult as it will lead to the use of microscopic instruments and sub-millimeter ports within the foreseeable future. The ultimate goal of MIT or MAT is noninvasive treatment with no instrument or ports being necessary. Although examples of this can be cited such as focused high intensity ultrasound for the ablation of hepatic tumours (tractless surgery), it is unlikely that this approach will have more than a limited application in the wide spectrum of the surgical disorders.

\section{Technology vs surgical skill}

The significant advances in MAT and MAS have been possible as a result of the combined influence of technological advances and skills of the operators. In this respect, the endoscopic operations and interventions that are performed today, and new procedures that are likely to come in the future, do not depend on technological advances alone; the latter facilitate the execution or make an operation possible. In the final analysis, safe and efficient execution is dependent on the unique skills of the surgeon or interventionalist. For example, the current MAT of localized coronary artery stenosis involves a radiological transvascular approach with dilatation of the stenosis and insertion of a stent to maintain luminal patency. Bypass surgery is required for more extensive coronary disease involving several vessels. Over the past few years a videothoracoscopic approach to coronary artery bypass surgery, avoiding median sternotomy has been introduced in a few pioneering centers. This significant development is clearly the outcome of unique surgical endoscopic skills based on the extensive experience of pioneering cardiac endo-surgeons. This example illustrates the fact that technology makes a procedure possible, but in the end it is the surgeon or interventional radiologist who must use the technology to execute the operation. Similar considerations apply to the need for conversion to open surgery during endoscopic surgery of the abdomen and chest. This is and always will be a fine balance between surgical experience, clinical judgment and available technology. 


\section{Future objectives of MIS/MAS}

The broad objectives which govern the future development of MAS interventions can be itemized as follows:

- Accurate and complete pre-surgical diagnosis

- On-line tomographic imaging of the operative tissue volume

- Presurgical planning of procedure and access trajectories

- Further minimization of the access to the operative site

- Improved technology for in-situ ablation and tissue approximation

Aside from the imaging system (Hopkin's rod lens telescope and CCD camera), most of the current instrumentation used in MAS has been developed by pioneers with technological knowledge and expertise in endoscopic surgery such as Wittmoser, Semm, Buess etc., working in close co-operation with industry. This association from the early design stage to the final prototype and its evaluation is crucial and, if anything, will assume greater importance in the R\&D of sophisticated technologies and imaging systems in the future. There is another factor. It seems likely with the introduction TES that complex technologies that are currently used by disciplines other than surgery, e.g., tomographic imaging by open MRI and hybrid fluoroscopy-spiral CT system, will be increasingly used in the future. Obviously expectations that surgeons in the next century would be required to be totally familiar with surgical tomography and advanced techniques for in-situ tissue ablation is unrealistic. As procedures become more complex requiring sophisticated imaging and technology, expert therapeutic intervention will necessitate a multidisciplinary team consisting of surgeons, radiologists, flexible endoscopists and physicists working together on specific disorders in accordance with their clinical interests-disease-related treatment groups. A patient with a specific disorder will be assessed by this team and the best MAT option depending on nature, stage of the disease and general condition will be determined by the group. In this fashion the optimal interventional therapeutic approach would be individualized to the patient's needs.

The co-operation with industry in technological and imaging Research and Development (R\&D) works both ways. For a productive association, the scientists working in industry and higher research institutions must understand the concepts behind the intended therapeutic advances. This requires some basic knowledge of the medical and surgical principles involved. In practice, this translates to whether the two parties can communicate and understand each other. The only valid practical approach to this problem is education of clinicians involved in R\&D in the basic principles of high technology related to MAT development concurrent with medical instruction of scientists, engineers and technologists involved in this co-operative research. Joint regular workshops on 'Surgery meets Technology' and information in journals such as the Technology Section introduced in this and other journals are obvious ways of achieving this mutual understanding.

\section{Tomographic Endosurgery (TEM)}

The first session of the new Technology Section of Surgical Endoscopy deals with the clinical results and technical data of tomographic interventions and future aspects of tomographic endosurgery [6]. Tomographic guidance and endoscopic surgery have to be regarded as complementary technologies. The endoscope provides a clear sharp colored image of the surface features of the operative field, whereas the on-line tomographic imaging provides data on the sub-surface nature of the entire operative tissue volume of the same operative field. This dual imaging yields the most comprehensive information of the anatomy, parenchymal details and intra-parenchymal pathology of all the organs and structures contained within the operating zone. In addition, the on-line tomographic information provides accurate information of the access trajectories of the instruments used for the intervention in real time and, if frameless stereotaxis is deployed, the exact position of the point of the instrument in space (x,y,z location) can be mapped on to the tomographic image of the operative tissue volume in real time. The precision and safety of the manipulations particularly in anatomically crowded and dangerous areas are thereby assured.

A further application of fast tomographic image guidance is in the field of surgical robotics and master slave manipulators that are under development by several groups [7-9]. All these systems are motorized and their effective control requires fail-safe systems in the as low as is reasonably possible (ALARP) region based on software guided by an external position control system, i.e., near real time tomographic imaging. The problem with such an image guided operating system (IGOS) is cost, and careful studies will be needed to establish the cost benefit to patient care with the introduction of these systems. If they convert a major MAS to a minimal MAS such that the procedure can be performed under local anaesthesia with immediate discharge, then specialized TES units equipped with this technology will flourish. As shown by Dr. Seibel's review, spinal TES has already reached this stage. Indeed, it has come of age.

\section{References}

1. Wickham J. Editorial. Min Invas Ther \& Allied Tech 1996: 1

2. Cuschieri A, Buess G. Nature and scope of endoscopic surgery. In: Operative Manual of Endoscopic Surgery, Cuschieri A, Buess G, Perissat J (eds) 1992; Springer: Berlin, pp 9-13

3. Cuschieri A, Crosthwaite G, Shimi S, Pietrabissa, Joypaul V, Tait I, Naziri W. Hepatic cryotherapy for liver tumours: development and clinical evaluation of a high-efficiency multineedle probe system for open and laparoscopic use. Surg Endosc 1995: 483-489

4. Buess G. IEM or what shall we call it? Min Invas Ther \& Allied Tech 1996: 1

5. Buess G. Why this journal? End Surg 1993: 1-2

6. Seibel R. Image guided minimal invasive therapy. Surg Endosc 1997: 154-162

7. Melzer A, Schurr MO, Kunert W, Buess G. Intelligent surgical instrument system. Concept and preliminary experimental application of components and prototypes. End Surg 1993: 165-170

8. Satava R, Simon LB. Teleoperation, telerobotics, and telepresence surgery. End Surg 1993: 151-153

9. Schurr MO, Melzer A, Kunert W, Dautzenberg P, Neisius B, Bretwieser W, Buess G. Experimental evaluation of components of an endoscopic manipulator system Artemis. October 1989, SMIT 6th International Meeting, Berlin

\section{A. Cuschieri}

Department of Surgery

Ninewalls Hospital and Medical School

Dundee, United Kingdom

\section{A. Melzer}

University Witten/Heidedre

Schulstrasse 10

Mulheim, Germany 